\title{
On the Pre-Log of Gaussian Fading Relay Channels
}

\author{
Tobias Koch \\ Signal and Information Processing Laboratory \\ Swiss Federal Institute of Technology (ETH) Zurich \\ CH-8092 Zurich, Switzerland \\ Email: tkoch@isi.ee.ethz.ch
}

\author{
Gerhard Kramer \\ Bell Laboratories, Lucent Technologies \\ 600 Mountain Ave, Murray Hill, NJ 07974 \\ Email: gkr@bell-labs.com
}

\begin{abstract}
The capacity of additive white Gaussian noise relay channels under Gaussian fading is investigated. The transmitter, the relay, and the receiver are all considered to be ignorant of the fading realizations. Capacity upper and lower bounds are derived with focus on the capacity pre-log, i.e., the limiting ratio of the capacity to the logarithm of the signal-to-noise ratio. Conditions are presented under which the upper and lower bounds on the capacity pre-log coincide.
\end{abstract}

KEYWORDS: Channel capacity, relay channels, Gaussian fading, non-coherent, capacity pre-log, fading number.

\section{INTRODUCTION}

Consider the relay channel depicted in Figure 1. To keep the model simple, we assume that the system is synchronous in the sense that the input and output symbols occur at the same time, and there is a small delay before the output symbols are forwarded to their terminals. We further assume that the input symbol $X_{2 k}$ is a function of $Y_{2}^{k-1}$, the input sequence $X_{1}^{n}$ is a function of the message $W$, and the estimate $\hat{W}$ of $W$ is a function of $Y_{3}^{n}$. Here, we use the notation $X_{1}^{n}$ to denote the sequence $\left(X_{11}, \ldots, X_{1 n}\right)$. Note that the relay can transmit and receive at the same time.

Let $\mathbb{C}$ be the set of complex numbers, and suppose that $X_{1 k} \in \mathbb{C}$ and $X_{2 k} \in \mathbb{C}$ are transmitted at time $k$. The time- $k$ channel outputs $Y_{2 k}$ and $Y_{3 k}$ are

$$
\begin{aligned}
& Y_{2 k}=H_{12 k} X_{1 k}+Z_{2 k} \\
& Y_{3 k}=H_{13 k} X_{1 k}+H_{23 k} X_{2 k}+Z_{3 k}
\end{aligned}
$$

where the processes $\left\{H_{12 k}\right\}_{k \in \mathbb{Z}},\left\{H_{13 k}\right\}_{k \in \mathbb{Z}},\left\{H_{23 k}\right\}_{k \in \mathbb{Z}}$, $\left\{Z_{2 k}\right\}_{k \in \mathbb{Z}}$, and $\left\{Z_{3 k}\right\}_{k \in \mathbb{Z}}$ are statistically independent of each other. Furthermore, the processes $\left\{H_{12 k}\right\}_{k \in \mathbb{Z}}$ and $\left\{Z_{2 k}\right\}_{k \in \mathbb{Z}}$ are independent of $\left\{X_{1 k}\right\}_{k \in \mathbb{Z}}$, but they might be dependent on $\left\{X_{2 k}\right\}_{k \in \mathbb{Z}}$, since the relay uses its past channel outputs to choose each $X_{2 k}$. All other processes are independent of both

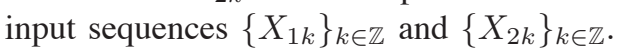

The additive noise terms $\left\{Z_{2 k}\right\}_{k \in \mathbb{Z}}$ and $\left\{Z_{3 k}\right\}_{k \in \mathbb{Z}}$ are sequences of independent and identically distributed (IID) circularly-symmetric, complex Gaussian random variables of zero mean and variance $\sigma^{2}>0$. We write this as $Z_{2 k} \sim$ $\mathcal{N}_{\mathbb{C}}\left(0, \sigma^{2}\right)$ and $Z_{3 k} \sim \mathcal{N}_{\mathbb{C}}\left(0, \sigma^{2}\right)$, where the notation $Z \sim$ $\mathcal{N}_{\mathbb{C}}\left(0, \sigma^{2}\right)$ indicates that the real and imaginary parts of $Z$ are independent and have a zero-mean Gaussian distribution of variance $\sigma^{2} / 2$.

The fading processes $\left\{H_{12 k}\right\}_{k \in \mathbb{Z}}, \quad\left\{H_{13 k}\right\}_{k \in \mathbb{Z}}$, and $\left\{H_{23 k}\right\}_{k \in \mathbb{Z}}$ are zero-mean, unit-variance, stationary, ergodic, circularly-symmetric, complex Gaussian processes with the respective spectral densities $f_{12}(\lambda), f_{13}(\lambda)$, and $f_{23}(\lambda)$, $-1 / 2 \leq \lambda \leq 1 / 2$. That is, for $t=1,2, r=2,3$, we have

$$
\begin{array}{ll}
\mathrm{E}\left[H_{t r(k+m)} H_{t r k}^{*}\right] & =\int_{-1 / 2}^{1 / 2} e^{j 2 \pi m \lambda} f_{t r}(\lambda) \mathrm{d} \lambda, k, m \in \mathbb{Z}, \\
\mathrm{E}\left[\left|H_{t r k}\right|^{2}\right] & =1,
\end{array}
$$

where $H_{t r k}^{*}$ denotes the complex conjugate of $H_{t r k}$. We further assume that the fading realizations are unknown to the transmitter, relay, and receiver. This channel model is also referred to as non-coherent.

We will study this channel under peak power constraints on the inputs, i.e., with probability one we have

$$
\left|X_{1 k}\right| \leq A_{1}, \quad\left|X_{2 k}\right| \leq A_{2}, \quad k \in \mathbb{Z}
$$

Additionally, we assume that, for some positive constant $\Upsilon$, we have

$$
\frac{A_{2}}{A_{1}}=\Upsilon .
$$

Suppose that the message $W$ is uniformly distributed on the set $\mathcal{W}=\left\{1,2, \ldots, e^{n R}\right\}$, and refer to $R$ as the rate. The rate $R$ is said to be achievable if there exist sequences $\left\{\pi_{n}(\cdot)\right\}_{n=1}^{\infty},\left\{\psi_{n}(\cdot)\right\}_{n=1}^{\infty}$ of mappings with $X_{1}^{n}=\phi_{n}(W)$ and $\hat{W}=\psi_{n}\left(Y_{3}^{n}\right)$ such that $\operatorname{Pr}[W \neq \hat{W}]$ tends to zero as $n$ goes to infinity. The capacity $C$ is the supremum of all achievable rates.

In this document we focus on the asymptotic capacity as $A_{1}$ (and hence also $A_{2}=\Upsilon A_{1}$ ) goes to infinity. In particular, we consider the capacity pre-log $\Pi$ defined as

$$
\Pi \triangleq \varlimsup_{A_{1} \rightarrow \infty} \frac{C\left(A_{1}\right)}{\log A_{1}^{2}}
$$

where $C\left(A_{1}\right)$ denotes the capacity under the peak power constraint $A_{1}$. Here, $\log (\cdot)$ is the natural logarithm function. All rates specified in this document are in nats per channel use. Lapidoth has shown [1] that the capacity pre-log of a point-to-point channel is given by

$$
\Pi=\mu(\{\lambda: f(\lambda)=0\})
$$

where $\mu(\cdot)$ denotes the Lebesgue measure on the interval $[-1 / 2,1 / 2]$, and $f(\lambda),-1 / 2 \leq \lambda \leq 1 / 2$, is the spectral density of the fading process. Note that the capacity pre-log of a point-to-point channel cannot be larger than $1 .{ }^{1}$ Further note

\footnotetext{
${ }^{1}$ It is shown below that the same is true for relay channels.
} 


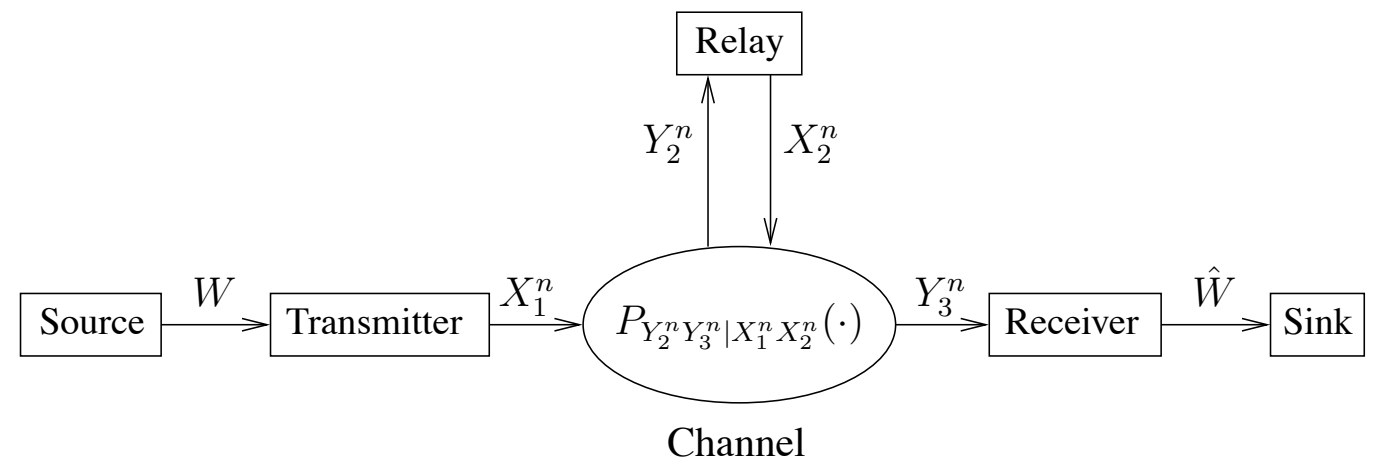

Fig. 1. The relay channel model.

that whenever the fading process is band-limited the pre-log is strictly larger than zero.

As we shall see, the pre-log depends on the mean-square error $\epsilon_{t r}^{2}\left(\delta^{2}\right)$ in predicting $H_{t r 0}$ based on the variance- $\delta^{2}$ noisy observation of the past $H_{t r(-1)}+W_{-1}, H_{t r(-2)}+W_{-2}, \ldots$ where $\left\{W_{k}\right\}_{k \in \mathbb{Z}}$ is a sequence of IID random variables with $W_{k} \sim \mathcal{N}_{\mathbb{C}}\left(0, \delta^{2}\right)$, and where $\left\{W_{k}\right\}_{k \in \mathbb{Z}}$ is independent of $\left\{H_{t r k}\right\}_{k \in \mathbb{Z}}$. That is, for $t=1,2, r=2,3$, we have (see [1, Section III])

$$
\epsilon_{t r}^{2}\left(\delta^{2}\right)=\exp \left\{\int_{-1 / 2}^{1 / 2} \log \left(f_{t r}(\lambda)+\delta^{2}\right) \mathrm{d} \lambda\right\}-\delta^{2} .
$$

Notice that it was proved by Lapidoth and Moser [2] that if the fading process of a point-to-point channel between two terminals $t$ and $r$ has a spectral density such that

$$
\lim _{\delta^{2} \downarrow 0} \epsilon_{t r}^{2}\left(\delta^{2}\right)>0
$$

then the corresponding capacity $C_{t r}(A)$ of this point-to-point channel grows only double-logarithmically in $A^{2}$, i.e.,

$$
\varlimsup_{A \rightarrow \infty}\left\{C_{t r}(A)-\log \log A^{2}\right\}<\infty
$$

and hence the pre-log is zero. We refer to processes that fulfill (10) as regular.

This paper is organized as follows. In the following two sections we derive upper and lower bounds on the capacity of the relay channel described above. In Section IV we use these bounds to compute bounds on the capacity pre-log. Section V addresses the asymptotic capacity when the fading processes are regular.

\section{AN UPPER BOUND}

An upper bound on the capacity $C\left(A_{1}\right)$ is given by

$$
\begin{array}{r}
C\left(A_{1}\right) \leq \lim _{n \rightarrow \infty} \frac{1}{n} \sup \min \left\{I\left(X_{1}^{n} ; Y_{2}^{n}, Y_{3}^{n}\right),\right. \\
\left.I\left(X_{1}^{n}, X_{2}^{n} ; Y_{3}^{n}\right)\right\} \\
\leq \min \left\{\lim _{n \rightarrow \infty} \frac{1}{n} \sup I\left(X_{1}^{n} ; Y_{2}^{n}, Y_{3}^{n}\right),\right. \\
\left.\lim _{n \rightarrow \infty} \frac{1}{n} \sup I\left(X_{1}^{n}, X_{2}^{n} ; Y_{3}^{n}\right)\right\}
\end{array}
$$

where the maximizations are over all input distributions satisfying the power constraints (5). Note that if the channel is memoryless, then equation (12) is one of the first steps in proving the max-flow min-cut upper bound (see [3, Theorem 14.7.1])

Since $X_{2 k}$ is a function of $Y_{2}^{k-1}$, one could use this causality relation to reduce the number of possible input distributions on $\left(X_{1}^{n}, X_{2}^{n}\right)$. However, we drop this restriction to get a capacity upper bound based on the point-to-point channels.

The first argument in the minimization in (13) can be upper bounded by the capacity of a single-input multiple-output fading channel with memory. Thus, we have (see [4, Theorem 5.14])

$$
\begin{aligned}
& \lim _{n \rightarrow \infty} \frac{1}{n} \sup I\left(X_{1}^{n} ; Y_{2}^{n}, Y_{3}^{n}\right) \\
& \leq \log \frac{1+\delta_{1}^{2}}{\epsilon_{12}^{2}\left(\delta_{1}^{2}\right)+\delta_{1}^{2}}+\log \frac{1+\delta_{1}^{2}}{\epsilon_{13}^{2}\left(\delta_{1}^{2}\right)+\delta_{1}^{2}}+\log \log \frac{A_{1}^{2}}{\sigma^{2}}+O(1)
\end{aligned}
$$

where $\delta_{1}^{2}=\sigma^{2} / A_{1}^{2}$ and where the $O(1)$ term is bounded in $A_{1}$. An upper bound on the second argument in the minimization in (13) is stated in the following theorem.

Theorem 1 For the relay channel described in Section I the mutual information $\lim _{n \rightarrow \infty} \frac{1}{n} \sup I\left(X_{1}^{n}, X_{2}^{n} ; Y_{3}^{n}\right)$ can be upper bounded as

$$
\begin{aligned}
& \lim _{n \rightarrow \infty} \frac{1}{n} \sup I\left(X_{1}^{n}, X_{2}^{n} ; Y_{3}^{n}\right) \\
& \leq \log \frac{1}{\epsilon_{\min }^{2}\left(\delta_{*}^{2}\right)}+\log \log \left(1+\frac{A_{1}^{2}}{\sigma^{2}}+\frac{A_{2}^{2}}{\sigma^{2}}\right)+O(1)
\end{aligned}
$$

where $\epsilon_{\min }^{2}\left(\delta_{*}^{2}\right)=\min \left\{\epsilon_{13}^{2}\left(\delta_{*}^{2}\right), \epsilon_{23}^{2}\left(\delta_{*}^{2}\right)\right\}$ and

$$
\delta_{*}^{2}=\min \left\{\delta_{2}^{2}, \delta_{3}^{2}\right\}, \quad \delta_{2}^{2}=\frac{\sigma^{2}}{2 A_{1}^{2}}, \quad \delta_{3}^{2}=\frac{\sigma^{2}}{2 A_{2}^{2}} .
$$

Proof: The proof is similar to the proof of the capacity upper bound derived in [5]. 
Combining (14) and (15), we obtain the final upper bound

$$
\begin{aligned}
& C\left(A_{1}\right) \leq \min \left\{\log \frac{1}{\epsilon_{\min }^{2}\left(\delta_{*}^{2}\right)}+O\left(\log \log A_{1}^{2}\right)\right. \\
& \left.\log \frac{1+\delta_{1}^{2}}{\epsilon_{12}^{2}\left(\delta_{1}^{2}\right)+\delta_{1}^{2}}+\log \frac{1+\delta_{1}^{2}}{\epsilon_{13}^{2}\left(\delta_{1}^{2}\right)+\delta_{1}^{2}}+O\left(\log \log A_{1}^{2}\right)\right\}
\end{aligned}
$$

where $O\left(\log \log A_{1}^{2}\right)$ grows at most double-logarithmically in $A_{1}^{2}$, and where $\delta_{1}^{2}$ and $\delta_{*}^{2}$ are given by (14) and (16), respectively.

\section{A LOWER BOUND}

A lower bound on $C\left(A_{1}\right)$ can be found by turning the relay off, i.e., set $X_{2 k}=0, k \in \mathbb{Z}$. In this case, we can achieve the capacity of the point-to-point channel between the transmitter and receiver. Thus, we have (see $[1$, Section V])

$$
C\left(A_{1}\right) \geq \log \frac{1}{\epsilon_{13}^{2}\left(\xi_{1}^{2}\right)+\frac{2}{5} \xi_{1}^{2}}+O(1)
$$

with $\xi_{1}^{2}=4 \sigma^{2} / A_{1}^{2}$.

Another lower bound can be derived by using a decodeand-forward (DF) strategy. One can show that if $X_{1}^{n}$ and $X_{2}^{n}$ are statistically independent, then DF yields

$$
\begin{aligned}
& C\left(A_{1}\right) \\
& \geq \lim _{n \rightarrow \infty} \frac{1}{n} \sup \min \left\{I\left(X_{1}^{n} ; Y_{2}^{n} \mid X_{2}^{n}\right), I\left(X_{1}^{n}, X_{2}^{n} ; Y_{3}^{n}\right)\right\} \\
& =\lim _{n \rightarrow \infty} \frac{1}{n} \sup \min \left\{I\left(X_{1}^{n} ; Y_{2}^{n}\right), I\left(X_{1}^{n}, X_{2}^{n} ; Y_{3}^{n}\right)\right\}
\end{aligned}
$$

where the maximizations are over all product input distributions $P_{X_{1}^{n}(\cdot)} \cdot P_{X_{2}^{n}}(\cdot)$ on $\left(X_{1}^{n}, X_{2}^{n}\right)$ satisfying (5). The equality in (20) follows because we choose $X_{1}^{n}$ and $X_{2}^{n}$ to be independent, and because $Y_{2}^{n}$ is a noisy function of $X_{1}^{n}$ only. Note that choosing $X_{1}^{n}$ and $X_{2}^{n}$ to be dependent, (19) generalizes the DF rates of Cover and El Gamal [6, Theorem 1] to channels with memory.

The rate on the right-hand side of (19) can be achieved using block Markov superposition encoding where transmission is performed in $B$ blocks of length $n$. Note that here we are generating blocks of sequences $X_{2}^{n}$ by using only past blocks of sequences $Y_{2}^{n}$. Thus, the coding strategy does not use feedback immediately. This property lets us maximize the minimum in (20) over all input distributions $P_{X_{1}^{n}}(\cdot) \cdot P_{X_{2}^{n}}(\cdot)$ without considering a causality constraint.

The following theorem establishes a lower bound on (20).

Theorem 2 Let $\left\{X_{1 k}\right\}_{k \in \mathbb{Z}}$ and $\left\{X_{2 k}\right\}_{k \in \mathbb{Z}}$ be sequences of IID random variables, independent of each other, and with

$$
\begin{array}{ll}
X_{1 k} \sim \mathcal{U}\left(\left\{x \in \mathbb{C}: \frac{A_{1}^{\alpha}}{2} \leq|x| \leq A_{1}^{\alpha}\right\}\right), & k \in \mathbb{Z} \\
& 0<\alpha \\
X_{2 k} \sim \mathcal{U}\left(\left\{x \in \mathbb{C}: \frac{A_{2}}{2} \leq|x| \leq A_{2}\right\}\right), & k \in \mathbb{Z}
\end{array}
$$

where $\mathcal{U}(\mathcal{S})$ denotes the uniform distribution over the set $\mathcal{S}$. Then, the right-hand side of (20) can be lower bounded by

$$
\begin{aligned}
& \lim _{n \rightarrow \infty} \frac{1}{n} \sup \min \left\{I\left(X_{1}^{n} ; Y_{2}^{n}\right), I\left(X_{1}^{n}, X_{2}^{n} ; Y_{3}^{n}\right)\right\} \\
& \geq \sup _{0<\alpha<1} \min \left\{\begin{array}{c}
\log \frac{1}{\epsilon_{12}^{2}\left(\xi_{2}^{2}\right)+\frac{2}{5} \xi_{2}^{2}}+O(1), \\
\log \frac{1}{\epsilon_{23}^{2}\left(\xi_{3}^{2}\right)+\frac{2}{5} \xi_{3}^{2}}+O(1)
\end{array}\right\}
\end{aligned}
$$

with

$$
\xi_{2}^{2}=\frac{4 \sigma^{2}}{A_{1}^{2 \alpha}}, \quad \xi_{3}^{2}=\frac{4 A_{1}^{2 \alpha}}{A_{2}^{2}}+\frac{4 \sigma^{2}}{A_{2}^{2}} .
$$

Proof: We omit the proof due to its length. The basic idea is the following. The arguments in the minimization in (20) can be viewed as the achievable rates of a single-input singleoutput fading channel with memory, and of a multiple-input single-output fading channel with memory, respectively. The choice of (21) and (22) is motivated by the asymptotic analysis of these two channels performed in [1] and [4]. Lapidoth has shown [1] that the capacity pre-log of the former channel can be achieved by inputs that are distributed according to (22). It was proved in [4, Corollary 5.13] that the pre-log of the latter channel can be achieved by transmitting from only one antenna, namely the one that yields the largest pre-log. Thus, one channel input is distributed according to (22) whereas the other inputs remain silent. This corresponds to a switch-off of either the relay or the transmitter. Since the former case is already considered in (18), we focus on the latter. By turning the transmitter off the rate (20) is zero. Instead, we choose $P_{X_{1}^{n}}(\cdot) \cdot P_{X_{2}^{n}}(\cdot)$ such that $\lim _{n \rightarrow \infty} \frac{1}{n} I\left(X_{1}^{n} ; Y_{2}^{n}\right)$ is as large as possible, but where the ratio $X_{1 k} / X_{2 k}$ tends to zero as $A_{1}$ and $A_{2}$ go to infinity. This will have a similar effect on $\lim _{n \rightarrow \infty} \frac{1}{n} I\left(X_{1}^{n}, X_{2}^{n} ; Y_{3}^{n}\right)$ as turning the transmitter off when $A_{1}$ becomes large. The proof of the lower bound (23) is then similar to that in $[1$, Section V].

Combining (18) and (23), we obtain the final lower bound

$$
\begin{gathered}
C\left(A_{1}\right) \\
\log \frac{1}{\epsilon_{13}^{2}\left(\xi_{1}^{2}\right)+\frac{2}{5} \xi_{1}^{2}}+O(1), \\
\left.\sup _{0<\alpha<1} \min \left\{\begin{array}{c}
\log \frac{1}{\epsilon_{12}^{2}\left(\xi_{2}^{2}\right)+\frac{2}{5} \xi_{2}^{2}}+O(1), \\
\log \frac{1}{\epsilon_{23}^{2}\left(\xi_{3}^{2}\right)+\frac{2}{5} \xi_{3}^{2}}+O(1)
\end{array}\right\}\right\}
\end{gathered}
$$

where $\xi_{1}^{2}, \xi_{2}^{2}$, and $\xi_{3}^{2}$ are given in (18) and (24), respectively. Note that (25) aims at a good characterization of the capacity pre-log and may be a poor lower bound on the capacity. Indeed, if, for example, the fading processes are regular, i.e.,

$$
\lim _{\delta^{2} \downarrow 0} \epsilon_{t r}^{2}\left(\delta^{2}\right)>0, \quad t=1,2, \quad r=2,3,
$$

then the lower bound (25) is bounded in $A_{1}$ even though $C\left(A_{1}\right)$ is not. 


\section{THE PRE-LOG}

Let $\Pi_{t r}$ denote the capacity pre-log of the point-to-point channel between the terminals $t$ and $r$. With the aid of (17) and (25), we obtain the following bounds on $\Pi$.

Corollary 3 The capacity pre-log of the channel described in Section I is upper bounded by

$$
\Pi \leq \min \left\{\Pi_{12}+\Pi_{13}, \max \left\{\Pi_{13}, \Pi_{23}\right\}\right\}
$$

and lower bounded by

$$
\Pi \geq \max \left\{\Pi_{13}, \sup _{0<\alpha<1} \min \left\{\alpha \Pi_{12},(1-\alpha) \Pi_{23}\right\}\right\} .
$$

Proof: The bounds (27) and (28) follow by an asymptotic analysis of the capacity upper (17) and lower bounds (25) similar to that in [1, Section VIII].

If either $\Pi_{12}=0$, or $\Pi_{13} \geq \Pi_{23},{ }^{2}$ then the bounds (27) and (28) coincide and yield

$$
\Pi=\Pi_{13} .
$$

This pre-log can be achieved without making use of the relay. Note that the condition $\Pi_{13} \geq \Pi_{23}$ includes also the case $\Pi_{23}=0$ since the pre-log cannot be negative.

When the pre-logs $\Pi_{12}$ and $\Pi_{23}$ are strictly larger than zero, then $\min \left\{\alpha \Pi_{12},(1-\alpha) \Pi_{23}\right\}$ is maximized by

$$
\alpha=\frac{\Pi_{23}}{\Pi_{12}+\Pi_{23}}
$$

and (28) reduces to

$$
\Pi \geq \max \left\{\Pi_{13}, \frac{\Pi_{12} \Pi_{23}}{\Pi_{12}+\Pi_{23}}\right\} .
$$

For situations where the bounds (27) and (28) do not coincide, i.e., when $\Pi_{12}>0, \Pi_{23}>0$, and $\Pi_{13}<\Pi_{23}$, the difference $\Delta$ between the upper (27) and lower bounds (28) is

$$
\begin{aligned}
\Delta & =\min \left\{\Pi_{12}+\Pi_{13}, \Pi_{23}\right\}-\max \left\{\Pi_{13}, \frac{\Pi_{12} \Pi_{23}}{\Pi_{12}+\Pi_{23}}\right\} \\
& =\min \left\{\Pi_{12}, \Pi_{23}-\Pi_{13}, \Pi_{13}+\frac{\Pi_{12}^{2}}{\Pi_{12}+\Pi_{23}}, \frac{\Pi_{23}^{2}}{\Pi_{12}+\Pi_{23}}\right\} .
\end{aligned}
$$

It can be shown numerically that $\Delta$ is at most 0.6173 . This difference arises when $\Pi_{12}=0.62, \Pi_{13}=0.38$, and $\Pi_{23}=1$.

To illustrate the above results, the capacity upper (17) and lower bounds (25) for two relay channels with different fading processes are depicted in Figures 2 and 3. Note that, for sufficiently large $A_{1}$, all bounds grow logarithmically in $A_{1}^{2}$ with slopes given by (27) and (31), respectively.

The upper and lower bounds plotted in Figure 2 correspond to a channel where the fading processes are such that $\Pi_{12}=$ $0.9, \Pi_{13}=0.7$, and $\Pi_{23}=0.5$. One can see that in this case the slopes of the upper and lower bounds are equal, and that

\footnotetext{
${ }^{2}$ This occurs when the fading realizations between the transmitter and receiver can be estimated more precisely than the fading realizations between the relay and receiver.
}

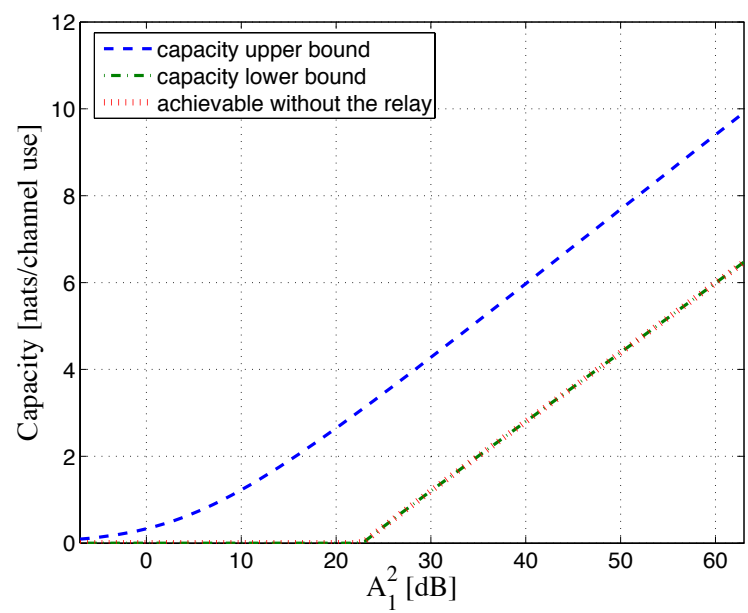

Fig. 2. Capacity upper (17) and lower bound (25) for $\Upsilon=1$ and $\sigma^{2}=1$. The fading processes have $\Pi_{12}=0.9, \Pi_{13}=0.7$, and $\Pi_{23}=0.5$.

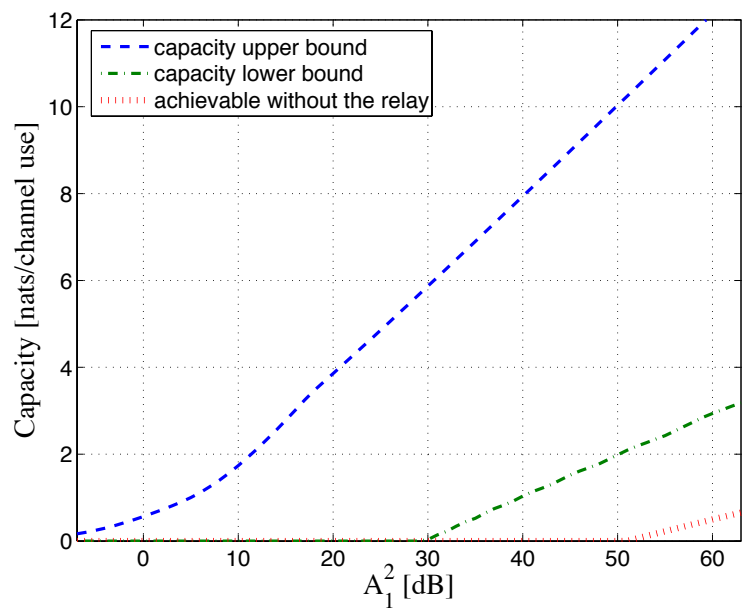

Fig. 3. Capacity upper (17) and lower bound (25) for $\Upsilon=1$ and $\sigma^{2}=1$. The fading processes have $\Pi_{12}=0.8, \Pi_{13}=0.2$, and $\Pi_{23}=0.9$.

the lower bound coincides with the curve corresponding to the rates achievable over the point-to-point channel between the transmitter and receiver. Thus, the capacity pre-log of the relay channel can be achieved without making use of the relay. This is in agreement with (29).

The upper and lower bounds plotted in Figure 3 correspond to a channel where $\Pi_{12}=0.8, \Pi_{13}=0.2$, and $\Pi_{23}=0.9$. It can be seen that here the slopes of the upper and lower bound differ from each other. We also observe that the slope of the curve corresponding to the rates that can be achieved without using the relay is smaller than the slope of the capacity lower bound. Thus, the relay helps to improve the pre-log.

Finally, we remark that the gaps between the capacity upper and lower bounds in Figures 2 and 3 are substantial. This suggests that much better bounds on the capacity can yet be found. 


\section{Additional Results}

For non-coherent point-to-point fading channels, it was shown by Lapidoth and Moser that when the fading process is regular in the sense that the present fading realization cannot be predicted precisely from its past, then the capacity grows double-logarithmically in the signal-to-noise ratio [2]. The same is true for relay channels. Thus, for these channels the capacity pre-log is always zero and it is more interesting to study the fading number introduced in [2]. For the relay channel depicted in Figure 1, the fading number is defined as

$$
\chi \triangleq \varlimsup_{P_{1} \rightarrow \infty}\left\{C\left(P_{1}\right)-\log \log P_{1}\right\}
$$

where $C\left(P_{1}\right)$ denotes the capacity under the average block power constraint $P_{1}$. That is,

$$
\begin{aligned}
& \frac{1}{n} \sum_{k=1}^{n} \mathrm{E}\left[\left|X_{1 k}\right|^{2}\right] \leq P_{1}, \\
& \frac{1}{n} \sum_{k=1}^{n} \mathrm{E}\left[\left|X_{2 k}\right|^{2}\right] \leq P_{2}
\end{aligned}
$$

where we assume that, for some positive constant $\Upsilon$, we have

$$
\frac{P_{2}}{P_{1}}=\Upsilon .
$$

We prove the following result.

Theorem 4 Consider the relay channel described in Section I under the average block power constraints (34) and (35). Furthermore, let the fading processes $\left\{H_{12 k}\right\}_{k \in \mathbb{Z}},\left\{H_{13 k}\right\}_{k \in \mathbb{Z}}$, and $\left\{H_{23 k}\right\}_{k \in \mathbb{Z}}$ be regular, i.e., the prediction errors $\epsilon_{12}^{2}(0)$, $\epsilon_{13}^{2}(0)$, and $\epsilon_{23}^{2}(0)$ are positive. Then, we have the following bounds on the fading number:

$$
\min \left\{\begin{array}{c}
-2 \gamma+\log \frac{1}{\epsilon_{12}^{2}(0)}+\log \frac{1}{\epsilon_{13}^{2}(0)}, \\
\max \left\{-1-\gamma+\log \frac{1}{\epsilon_{13}^{2}(0)},-1-\gamma+\log \frac{1}{\epsilon_{23}^{2}(0)}\right\}
\end{array}\right\}
$$

and

$$
\max \left\{\begin{array}{c}
-1-\gamma+\log \frac{1}{\epsilon_{13}^{2}(0)}, \\
\sup _{\substack{0<\alpha<\beta \\
\alpha<\beta<1}} \min \left\{\begin{array}{c}
-1-\gamma+\log \frac{1}{\epsilon_{12}^{2}(0)}+\log \alpha, \\
-1-\gamma+\log \frac{1}{\epsilon_{23}^{2}(0)}+\log (1-\beta)
\end{array}\right\}
\end{array}\right.
$$

where $\gamma \approx 0.577$ denotes Euler's constant.

Proof: The upper bound is proved in [5]. The proof of the lower bound is similar to the proof of the capacity lower bound (25).

If $\epsilon_{13}^{2}(0) \leq \epsilon_{23}^{2}(0)$, then the bounds (37) and (38) coincide and yield an exact expression of the fading number. In this case the fading number is

$$
\chi=-1-\gamma+\log \frac{1}{\epsilon_{13}^{2}(0)}
$$

and can be achieved without making use of the relay. Note that the relay can be beneficial, i.e., the fading number of the relay channel can be higher than the fading number of the point-to-point channel between the transmitter and receiver. For example, consider the situation where $\epsilon_{13}^{2}(0)=1$ and $\epsilon_{12}^{2}(0)=\epsilon_{23}^{2}(0)=1 / 8$. In this case, the bounds (37) and (38) yield the respective

$$
\chi \leq-1-\gamma+\log 8
$$

and

$$
\begin{aligned}
\chi & \geq-1-\gamma+\log 8+\log \frac{1}{2} \\
& =-1-\gamma+\log 4
\end{aligned}
$$

where we used the fact that in our example the supremum in (38) is achieved by $\alpha=\beta=1 / 2$. The point-to-point channel between the transmitter and the receiver provides only a fading number of $-1-\gamma$.

\section{ACKNOWLEDGMENT}

Discussions with Amos Lapidoth are gratefully acknowledged.

\section{REFERENCES}

[1] A. Lapidoth, "On the asymptotic capacity of stationary Gaussian fading channels," IEEE Transactions on Information Theory, vol. 51, no. 2, pp. 437-446, February 2005.

[2] A. Lapidoth and S. M. Moser, "Capacity bounds via duality with applications to multiple-antenna systems on flat fading channels," IEEE Transactions on Information Theory, vol. 49, no. 10, pp. 2426-2467, October 2003.

[3] T. M. Cover and J. A. Thomas, Elements of Information Theory. John Wiley \& Sons, 1991.

[4] T. Koch, "On the asymptotic capacity of multiple-input single-output fading channels with memory," Master's thesis, Signal and Information Processing Laboratory, ETH Zurich, Switzerland, April 2004.

[5] T. Koch and G. Kramer, "Communication rates for noncoherent Gaussian relay channels," Bell Labs Technical Memorandum, ITD-04-45907Z, November 2004.

[6] T. M. Cover and A. A. El Gamal, "Capacity theorems for the relay channel," IEEE Transactions on Information Theory, vol. 25, no. 5, pp. 572-584, September 1979. 